\title{
Correspondence
}

\section{Psychological therapies for bipolar disorder - adjunct not alternative to pharmacological treatments}

The British Psychological Society (BPS) recently published a report, Understanding Bipolar Disorder- Why Some People Experience Extreme Mood Swings and What Can Help'.1 In the foreword they have clarified that the purpose of the report is to provide an overview of current knowledge about the disorder with a special emphasis on the psychological aspects. The authors hope that this report will become an important source of information for everyone and services would be tailored as per their recommendations.

From the outset, the report assumes an anti-psychiatry flavour. The authors have strong views about labelling extreme mood swings as an illness or treating them primarily with medications. They also cast serious doubts about the reliability and validity of psychiatric diagnoses by selectively using the personal anecdotes and evidence from the literature. We would agree with some of their statements and concur that the psychiatric diagnoses are not perfect, but they are based on scientific data about the cluster of symptoms, genetics and presumed aetiology, course and outcome and response to treatment. Furthermore, the arguments put forward can also be applied to many chronic physical health problems such as diabetes, hypertension, etc. However, the authors do not offer any alternatives to the diagnostic systems except that we should asses the degree to which a person is able to regulate his or her mood or behaviour. The running theme of the document is that bipolar disorder is a lifestyle choice and most individuals can control it or can be helped to control their mood swings by psychological therapies. What is shocking is that the authors make these sweeping statements without giving any evidence to support them. They have selectively used the evidence to vindicate their stand while turning a blind eye to other evidence; likewise, at times they have completely misconstrued the available evidence. For example, throughout the report the emphasis has been on the effectiveness of psychological therapy; all the research cited has been done on patients who were on medications, either stable or in a depressed state. We are not aware of any study which was done on either drug-naive or manic patients. The authors have also ignored the evidence that did not suit them. Scott et $a l^{2}$ conducted a large, multicentre randomised controlled trial and compared treatment as usual with cognitive-behavioural therapy (CBT) and found no beneficial effect of CBT. Moreover, the authors of the BPS report also did not mention that one of the proposed mechanisms for the effectiveness of psychological therapies is by improving adherence to medications. ${ }^{3,4}$ Therefore, the only conclusion that can be drawn from the available evidence is that psychological therapies, if used in conjunction with the pharmacological therapies, can enhance functional and symptomatic outcomes of bipolar disorder. ${ }^{5}$

The BPS was one of the contributors to the National Institute for Health and Clinical Excellence (NICE) guideline on bipolar disorder, but their current document is at odds with the NICE recommendations. Most guidelines recommend psychological therapy along with pharmacological treatment, not in place of it. Therefore, in its current form the document is misleading and is more an opinion piece than scientific publication.

1 British Psychological Society. Understanding Bipolar Disorder - Why Some People Experience Extreme Mood Swings and What Can Help. British Psychological Society, 2010.

2 Scott J, Paykel E, Morriss R, Bentall R, Kinderman P, Johnson T, et al. Cognitive-behavioural therapy for severe and recurrent bipolar disorders. Randomised controlled trial. Br J Psychiatry 2006; 188 313-20.

3 Lam D. What can we conclude from studies on psychotherapy in bipolar disorder? Invited commentary on . . . Cognitive-behavioural therapy for severe and recurrent bipolar disorders. Br J Psychiatry 2006; 188: 321-2.

4 Miklowitz DJ, Scott J. Psychosocial treatments for bipolar disorder: cost-effectiveness, mediating mechanisms, and future directions. Bipolar Disord 2009; 11 (suppl 2): 110-22.

5 Milkowitz D. Adjunctive psychotherapy for bipolar disorder: state of the evidence. Am J Psychiatry 2008; 165: 1408-19.

Sumeet Gupta, consultant psychiatrist, Tees, Esk and Wear Valleys NHS Foundation Trust, Hundens Lane Resource Centre, Darlington, email: Sumeet.Gupta@tewv.nhs.uk; Johanna Brown, core trainee CT2, Hundens Lane Resource Centre.

doi: $10.1192 / p b .35 .5 .196$

\section{The Recovery Star: is it a valid tool?}

As a clinical psychologist working in an in-patient psychiatric setting, I am fully supportive of the principles behind the recovery model (or recovery approach) in the treatment of severe mental health problems. As such, I am fully supportive of efforts to ensure that the recovery approach is at the heart of the service delivery.

I have observed that a number of services - including our own - have adopted the Recovery Star model. The model is 'a tool for supporting and measuring change when working with adults of working age who are accessing mental health support services' (www.mhpf.org.uk/recoveryStarApproach.asp). Although I am supportive of the aim to measure such change, I am concerned that the Recovery Star model itself does not appear to have been considered in any peer-reviewed publications. Furthermore, there do not appear to be any available normative data published alongside the instrument, or any statistics indicating its reliability and validity. Given that the authors specifically describe the tool as something to be used to measure change, this is a very notable omission. Without such data it is impossible to know whether, for example, two different scores on two different occasions represent genuine therapeutic change or simply arise out of error; nor is it possible to know the extent to which two different clinicians using the tool would be expected to concur with each other. Furthermore, the tool proposes that ten different factors of recovery exist, yet again there is no mention of a factor analysis suggesting how such factors were derived or how they interrelate.

Although the development of instruments to measure patients' perceptions of engagement in the recovery model is 